\title{
Drug resistance analysis of bacterial strains isolated from burn patients
}

\author{
L.F. Wang, J.L. Li, W.H. Ma and J.Y. Li \\ Inner Mongolia Institute of Burn Research, \\ The Third Affiliated Hospital of Inner Mongolia Medical University, Baotou, \\ Inner Mongolia, China \\ Corresponding author: L.F. Wang \\ E-mail:1fjycn@yeah.net
}

Genet. Mol. Res. 13 (4): 9727-9734 (2014)

Received September 10, 2013

Accepted November 11, 2013

Published January 22, 2014

DOI http://dx.doi.org/10.4238/2014.January.22.10

\begin{abstract}
This study aimed to analyze the spectrum and drug resistance of bacteria isolated from burn patients to provide a reference for rational clinical use of antibiotics. Up to 1914 bacterial strain specimens isolated from burn patients admitted to hospital between 2001 and 2010 were subjected to resistance monitoring by using the K-B paper disk method. Retrospective analysis was performed on drug resistance analysis of burn patients. The top eight bacterium strains according to detection rate. A total of 1355 strains of Gram-negative $\left(\mathrm{G}^{-}\right)$bacteria and 559 strains of Gram-positive $\left(\mathrm{G}^{+}\right)$bacteria were detected. The top eight bacterium strains, according to detection rate, were Acinetobacter baumannii, Pseudomonas aeruginosa, Staphylococcus aureus, Escherichia coli, Staphylococcus epidermidis, Klebsiella pneumoniae, Enterobacter cloacae, and Enterococcus. Drug resistance rates were higher than $90 \%$ in A. baumannii, P. aeruginosa, S. epidermidis, and $S$. aureus, which accounted for 52.2, 21.7, 27.8, and 33.3\%, respectively, of the entire sample. Those with drug resistance rates lower than $30 \%$ accounted for $4.3,30.4,16.7$, and 16.7\%, respectively. Multidrugresistant $S$. aureus (MRSA) and methicillin-resistant S. epidermidis (MRSE) accounted for 49.2 and $76.4 \%$ of the S. epidermis and S. aureus
\end{abstract}


resistance, respectively. Antibacterial drugs that had drug resistance rates to MRSE and MRSA higher than $90 \%$ accounted for 38.9 and $72.2 \%$, respectively, whereas those with lower than $30 \%$ drug resistance rates accounted for 11.1 and $16.7 \%$, respectively. The burn patients enrolled in the study were mainly infected with $\mathrm{G}^{-}$bacteria. These results strongly suggest that clinicians should practice rational use of antibiotics based on drug susceptibility test results.

Key words: Burns; Bacteria; Antibiotics; Drug resistance

\section{INTRODUCTION}

Anti-infection treatment is an important step during the treatment of burns, and antibiotic application is a crucial means of infection prevention and control. However, along with the widespread use of antibiotics, the bacterium spectrum and bacterial drug resistance have also developed; reinforced multidrug resistance in bacterium results in great difficulties in clinical treatment. Thus, accurate knowledge of the bacterium spectrum, bacterial resistance trend, and changes in drug resistance to antibiotics are essential for determining the rational clinical use of antibiotics ( $\mathrm{Li}$ et al., 2005a). In this study, the strains and respective drug resistances of bacteria isolated from burn patients admitted to our unit between January 2001 and December 2010 were retrospectively analyzed to provide useful data for determining the rational clinical use of antibiotics.

\section{MATERIAL AND METHODS}

\section{Bacterium source}

A total of 1914 bacterial strains were isolated from burn patients who were hospitalized between January 2001 and December 2012, including from wound secretions, blood, sputum, and other specimens. Up to 1669 wound secretion specimens (87.2\%), 185 sputum specimens $(9.7 \%), 53$ blood specimens $(2.8 \%)$, and 7 drainage fluid specimens $(0.4 \%)$ were obtained. This study was conducted in accordance with the Declaration of Helsinki.

\section{General methods}

According to the "National Guide to Clinical Laboratory Procedures", the bacteria were identified through analytical profile index system identification, adapted from BioMerienx (France). The results were determined based on the National Committee on Clinical Laboratory Standards (2000 edition). The K-B paper disk agar diffusion method was adopted in the drug sensitivity test. The quality control strains used were standard strains, including Escherichia coli ATCC25922, Staphylococcus aureus ATCC25923, and Pseudomonas aeruginosa ATCC27853. A portion of the antibacterial drug paper disks was obtained from the Oxiod Company (Basingstoke, Hampshire, England), including imipenem, cefepime, ceftazidime/ clavulanic acid, and cefotaxime/clavulanic acid. The remaining drug-sensitive paper disks were purchased from Beijing Tiantan Biological Products Co. Ltd. (Beijing, China). 


\section{Diagnostic criteria for drug resistance}

The diagnostic criteria for drug resistance were established in accordance with the guidelines set by the National Committee for Clinical Laboratory Standards.

\section{Statistical analysis}

Data were analyzed with the WHONET software, version 5.3.

\section{RESULTS}

\section{Bacterial separation rate}

Up to 1355 strains of gram-negative $\left(\mathrm{G}^{-}\right)$rod bacteria and 559 strains of gram-positive $\left(\mathrm{G}^{+}\right)$rod bacteria were detected, accounting for 70.8 and $29.2 \%$ of the sample, respectively. The separation rates of $\mathrm{G}^{-}$and $\mathrm{G}^{+}$rod bacteria in burn patients admitted to hospital between 2001 and 2010 are shown in Figure 1. The distribution of the 1914 bacterial strains is shown in Figure 2.

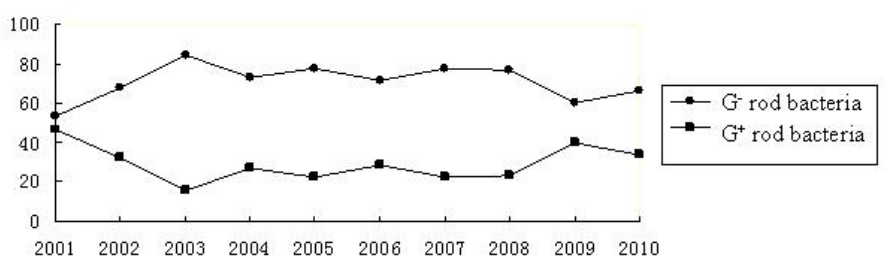

Figure 1. Separation rates of $\mathrm{G}^{-}$rod bacteria and $\mathrm{G}^{+}$rod bacteria (\%) during 2001-2010.

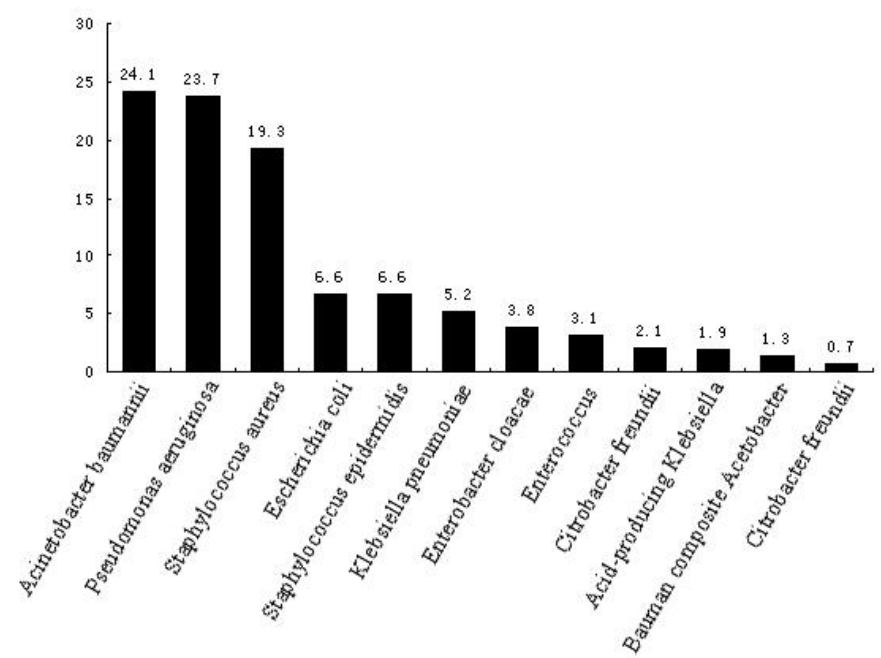

Figure 2. Distribution of 1914 bacterium strains. Note: MRSA was accounted for $76.4 \%$ in Staphylococcus aureus and MRSE was accounted for $49.2 \%$ in Staphylococcus epidermidis. 


\section{Bacterial detection rate}

According to the detection rate, the top eight bacteria were Acinetobacter baumannii, P. aeruginosa, S. aureus, E. coli, Staphylococcus epidermidis, Klebsiella pneumoniae, Enterobacter cloacae, and Enterococcus. The distribution of bacteria throughout the period of 2001 to 2010 is shown in Table 1.

\begin{tabular}{|c|c|c|c|c|c|c|c|c|c|}
\hline Years & Total & aba & pae & sau & eco & sep & kpn & ecl & ent \\
\hline 2001 & 32 & $0 / 0$ & $6 / 18.8$ & $1 / 3.1$ & $4 / 12.5$ & $10 / 31.3$ & $1 / 3.1$ & $5 / 15.6$ & $4 / 12.5$ \\
\hline 2002 & 78 & $8 / 10.3$ & $17 / 21.8$ & 15/19.2 & $12 / 15.4$ & $6 / 8.0$ & $4 / 5.1$ & $7 / 9.0$ & $2 / 2.6$ \\
\hline 2003 & 94 & $14 / 14.9$ & $31 / 33.0$ & $8 / 8.5$ & $5 / 5.3$ & $3 / 3.2$ & $17 / 18.1$ & $10 / 10.6$ & $2 / 2.1$ \\
\hline 2004 & 81 & $22 / 27.2$ & $17 / 21.0$ & $5 / 6.2$ & $7 / 8.6$ & $15 / 18.5$ & $8 / 9.9$ & $3 / 3.7$ & $2 / 2.5$ \\
\hline 2005 & 127 & $49 / 38.6$ & $20 / 15.7$ & $15 / 11.8$ & $9 / 7.1$ & $10 / 7.9$ & $3 / 2.4$ & $10 / 7.9$ & $2 / 1.6$ \\
\hline 2006 & 240 & $56 / 23.3$ & $58 / 24.2$ & $21 / 8.8$ & $29 / 12.1$ & $35 / 14.6$ & $12 / 5.0$ & $20 / 8.3$ & $13 / 5.4$ \\
\hline 2007 & 292 & $66 / 22.6$ & $58 / 19.9$ & $31 / 10.6$ & $17 / 5.8$ & $30 / 10.3$ & $16 / 5.5$ & $2 / 0.7$ & $4 / 1.4$ \\
\hline 2008 & 317 & $64 / 20.2$ & $147 / 46.4$ & $68 / 21.5$ & $5 / 1.6$ & $0 / 0$ & $3 / 0.9$ & $5 / 1.6$ & $6 / 1.9$ \\
\hline 2009 & 408 & $99 / 24.3$ & $77 / 18.9$ & $150 / 36.8$ & $18 / 4.4$ & $11 / 2.7$ & $27 / 6.6$ & $10 / 2.5$ & $2 / 0.5$ \\
\hline 2010 & 245 & $84 / 34.3$ & $22 / 9.0$ & $55 / 22.4$ & $21 / 8.6$ & $6 / 2.4$ & $17 / 6.9$ & $1 / 0.4$ & $22 / 9.0$ \\
\hline Total & 1914 & $462 / 24.1$ & $454 / 23.7$ & $369 / 19.3$ & $127 / 6.6$ & $126 / 6.6$ & $100 / 5.6$ & $73 / 3.8$ & $59 / 3.1$ \\
\hline
\end{tabular}

\section{Drug resistance of $\mathrm{G}^{-}$rod bacteria in the top eight bacterial strains according to detection rate}

The drug resistances of $\mathrm{G}^{-}$rod bacteria in the top eight bacteria according to detection rate are shown in Table 2. The antibacterial drugs with a drug resistance rate to $A$. baumannii lower than $30 \%$ only included cefoperazone/sulbactam, accounting for $4.3 \%$; those with drug resistance rates higher than $90 \%$ included ampicillin, aztreonam, SMZCo, chloramphenicol, piperacillin, gentamycin, cefoperazone, ceftriaxone, ceftazidime, cefazolin, tobramycin, and cefotaxime, accounting for $52.2 \%$. The antibacterial drugs with drug resistance rates to $P$. aeruginosa lower than $30 \%$ included amikacin, aztreonam, piperacillin/tazobactam, cefepime, cefoperazone/sulbactam, ceftazidime, and imipenem, accounting for $30.4 \%$; those with higher than $90 \%$ drug resistance rates included ampicillin, ampicillin/sulbactam, SMZCo, chloramphenicol, and cefazolin, accounting for $21.7 \%$.

\section{Drug resistance of $\mathrm{G}^{+}$rod bacteria}

The drug resistances of $\mathrm{G}^{+}$rod bacteria in the top eight bacteria according to detection rate are shown in Table 3. The antibacterial drugs with lower than $30 \%$ drug resistance rate to $S$. aureus and $S$. epidermidis accounted for $16.7 \%$; those with higher than a $90 \%$ drug resistance rate accounted for $33.3 \%$ and $27.8 \%$, respectively. The antibacterial drugs with a lower than $30 \%$ drug resistance rate to multidrug-resistant S. aureus (MRSA) and methicillin-resistant S. epidermidis (MRSE) accounted for 16.7 and $11.1 \%$, respectively, and those with higher than $90 \%$ drug resistance rate accounted for 72.2 and $38.9 \%$, respectively. 


\begin{tabular}{|c|c|c|c|c|c|c|}
\hline Antibiotics & $\mathrm{G}^{-}$ & aba & pae & eco & kpn & ecl \\
\hline Amikacin & $46.6 / 1119$ & $82.5 / 354$ & $24.6 / 414$ & $19.4 / 108$ & $25.3 / 99$ & $58.8 / 68$ \\
\hline Amoxicillin/clavulanic acid & $81.8 / 11$ & - & $75.0 / 4$ & $100 / 1$ & $0 / 1$ & $100 / 3$ \\
\hline Ampicillin & $96.3 / 408$ & $91.5 / 59$ & $93.9 / 33$ & $98.2 / 112$ & $97.9 / 96$ & $100 / 68$ \\
\hline Ampicillin/Shubatan & $82.5 / 63$ & $42.9 / 14$ & $100 / 37$ & $33.3 / 3$ & $100 / 3$ & $75.0 / 4$ \\
\hline Aztreonam & $54.2 / 1123$ & $90.8 / 358$ & $22.9 / 415$ & $57.8 / 109$ & $39.4 / 99$ & $63.2 / 68$ \\
\hline Cotrimoxazole & $90.0 / 448$ & $90.4 / 146$ & $93.8 / 210$ & $92.9 / 28$ & $52.4 / 21$ & $100 / 11$ \\
\hline Ciprofloxacin & $60.5 / 1106$ & $83.6 / 415$ & $38.9 / 350$ & $76.9 / 108$ & $22.4 / 98$ & $53.3 / 60$ \\
\hline Chloramphenicol & $87.5 / 48$ & $100 / 18$ & $95.8 / 71$ & $50.0 / 2$ & $0 / 1$ & - \\
\hline Piperacillin & $72.2 / 1170$ & $91.8 / 416$ & $53.4 / 410$ & $74.1 / 112$ & $50.5 / 97$ & $87.9 / 66$ \\
\hline Piperacillin/tazobactam & $19.3 / 765$ & $36.4 / 316$ & $10.8 / 287$ & $1.8 / 56$ & $0 / 57$ & $3.6 / 28$ \\
\hline Gentamicin & $80.8 / 1144$ & $91.3 / 413$ & $77.8 / 383$ & $86.5 / 111$ & $50.0 / 94$ & $83.6 / 67$ \\
\hline Cefepime & $49.1 / 1143$ & $85.9 / 417$ & $16.4 / 408$ & $59.6 / 99$ & $25.0 / 88$ & $32.3 / 65$ \\
\hline Cefuroxime sodium & $71.6 / 285$ & $88.9 / 36$ & $84.0 / 25$ & $70.3 / 74$ & $56.0 / 84$ & $80.6 / 36$ \\
\hline Cefoperazone & $71.2 / 1040$ & $95.4 / 410$ & $56.4 / 388$ & $37.7 / 69$ & $47.6 / 82$ & $61.4 / 44$ \\
\hline Cefoperazone/Shubatan & $6.4 / 469$ & $5.0 / 200$ & $3.8 / 130$ & $5.0 / 40$ & $0 / 45$ & $0 / 36$ \\
\hline Ceftriaxone & $71.0 / 572$ & $95.4 / 218$ & $58.3 / 84$ & $62.5 / 80$ & $45.2 / 84$ & $63.4 / 41$ \\
\hline Ceftazidime & $56.8 / 1180$ & $93.3 / 418$ & $21.8 / 404$ & $57.1 / 112$ & $37.4 / 99$ & $66.2 / 71$ \\
\hline Cefazolin & $80.7 / 394$ & $96.2 / 52$ & $95.7 / 23$ & $74.1 / 108$ & $58.4 / 89$ & $98.5 / 68$ \\
\hline Tobramycin & $80.8 / 532$ & $94.0 / 183$ & $75.2 / 318$ & $100 / 8$ & $0 / 9$ & $100 / 1$ \\
\hline Imipenem & $32.2 / 1142$ & $57.0 / 426$ & $22.6 / 424$ & $0 / 112$ & $2.0 / 98$ & $1.5 / 68$ \\
\hline Ofloxacin & $53.5 / 86$ & $80.0 / 5$ & $44.1 / 68$ & $100 / 5$ & $100 / 3$ & $80.0 / 5$ \\
\hline Levofloxacin & $40.6 / 668$ & $39.6 / 260$ & $60.8 / 186$ & $62.2 / 45$ & $8.6 / 58$ & $20.0 / 20$ \\
\hline Cefotaxime & $65.1 / 728$ & $90.8 / 217$ & $53.3 / 152$ & $58.9 / 112$ & $37.8 / 98$ & $70.4 / 71$ \\
\hline
\end{tabular}

Table 3. Drug resistance of $\mathrm{G}^{+}$rod bacteria in the first eight bacterial strains according to detection rate (Drug resistance rate \%/Detection number).

\begin{tabular}{|c|c|c|c|c|c|c|}
\hline Antibiotics & $\mathrm{G}^{+}$ & Sau & MRSA & Sep & MRSE & Ent \\
\hline Amikacin & $88.0 / 75$ & $83.3 / 30$ & $92.0 / 3$ & $90.9 / 44$ & $100 / 7$ & $100 / 1$ \\
\hline Azithromycin & $95.2 / 273$ & $95 / 220$ & $99.4 / 168$ & $96.2 / 53$ & $96.3 / 27$ & - \\
\hline Ampicillin & $67.8 / 90$ & $96.6 / 29$ & $100 / 20$ & $94.1 / 17$ & $100 / 12$ & $41.5 / 41$ \\
\hline Oxacillin & $76.6 / 320$ & $79.5 / 259$ & $99.5 / 206$ & $63.3 / 60$ & $100 / 38$ & $100 / 1$ \\
\hline Cotrimoxazole & $77.9 / 367$ & $79.2 / 268$ & $88.4 / 207$ & $73.7 / 95$ & $73.5 / 68$ & $100 / 4$ \\
\hline Erythromycin & $93.9 / 488$ & $94.4 / 354$ & $98.6 / 277$ & $96.7 / 92$ & $96.2 / 53$ & $84.6 / 39$ \\
\hline Ciprofloxacin & $79.4 / 383$ & $82.6 / 298$ & $96.3 / 240$ & $65.7 / 70$ & $70.0 / 40$ & $80.0 / 15$ \\
\hline Clindamycin & $83.7 / 454$ & $90.4 / 354$ & $97.5 / 276$ & $59.1 / 93$ & $71.7 / 53$ & $100 / 4$ \\
\hline Rifampicin & $36.2 / 469$ & $35.2 / 341$ & $42.5 / 268$ & $27.8 / 97$ & $48.2 / 56$ & $76.6 / 30$ \\
\hline Chloramphenico & $14.5 / 399$ & $9.0 / 312$ & $8.0 / 264$ & $35.0 / 60$ & $48.6 / 37$ & $37.5 / 24$ \\
\hline Norfloxacin & $71.4 / 7$ & $100 / 2$ & $100 / 2$ & $75.0 / 4$ & $75.0 / 4$ & $0 / 1$ \\
\hline Gentamicin & $78.5 / 362$ & $84.6 / 279$ & $96.8 / 219$ & $58.7 / 75$ & $57.4 / 47$ & $50.0 / 8$ \\
\hline Teicoplanin & $1.7 / 239$ & $0 / 200$ & $0 / 167$ & $0 / 17$ & $0 / 2$ & $19.0 / 21$ \\
\hline Cefazolin & $83.3 / 144$ & $91.1 / 112$ & $97.1 / 105$ & $54.8 / 31$ & $100 / 10$ & $100 / 1$ \\
\hline Vancomycin & $0.8 / 520$ & $0 / 361$ & $0 / 283$ & $0 / 102$ & $0 / 61$ & $7.4 / 54$ \\
\hline Ofloxacin & $82.6 / 23$ & $81.8 / 11$ & $90.0 / 10$ & $66.7 / 6$ & $66.7 / 6$ & $100 / 3$ \\
\hline Levofloxacin & $73.9 / 207$ & $79.6 / 162$ & $91.8 / 134$ & $56.8 / 37$ & $42.1 / 19$ & $50.0 / 6$ \\
\hline Penicillin G & $80.4 / 582$ & $81.5 / 427$ & $99.6 / 279$ & $96.0 / 101$ & $100 / 59$ & $43.1 / 51$ \\
\hline
\end{tabular}

\section{DISCUSSION}

Retrospective analysis was performed to identify the bacteria and their drug resistances isolated from burn patients in our burn department. The results showed that the bacteria spectrum has changed in the past 10 years, and bacteria show different degrees of resistance to antibiotics. From 2001 to 2010, 1914 bacterial strains were detected from burn patients, including from wound secretions, blood, sputum, and other specimens. The top eight bacteria were identified 
as A. baumannii (462 strains), P. aeruginosa (454 strains), S. aureus (369 strains, including 282 MRSA strains), E. coli (127 strains), S. epidermidis (126 strains, including 62 MRSE strains), K. pneumoniae (100 strains), E. cloacae (73 strains), and Enterococcus (59 strains). Twice as many $\mathrm{G}^{-}$rod bacteria $(70.8 \%)$ were detected as $\mathrm{G}^{+}$rod bacteria $(29.2 \%)$, which is a higher rate than any survey data reported prior to 2000 (Xu et al., 2002), but is similar to those reported after 2000 (Wei and Liu, 2006). These findings indicate that $\mathrm{G}^{-}$rod bacteria remain the dominant bacteria in burn infections in XX hospital. In the past three years, P. aeruginosa, S. aureus, and A. baumannii alternately occupied the top three places, which is similar to rankings previously cited both in China and elsewhere (Wei and Liu, 2006; Essayagh et al., 2011). Thus, the clinical treatment of burn infections should receive more attention.

Along with the widespread use of the three generations of cephalosporin, ceftazidime, and carbapenems, imipenem, the drug resistance rate units used in these drugs has noticeably increased; the more intractably conditioned pathogens are typically screened out under the pressure of antibiotics, which is almost the same for patients in burn wards and intensive care units (Xiao, 2004). Although new antibiotics are constantly being developed, bacterial drug resistance is strengthening, which causes difficulties in clinical treatment of burn infections. In this study, $\mathrm{G}^{-}$rod bacteria accounted for five of the top eight detected bacterial strains. $A$. baumannii was the most frequently detected, followed by P. aeruginosa. Among the detected $\mathrm{G}^{-}$rod bacteria from burn patients, $A$. baumannii exhibited an annually increasing trend that showed a higher detection rate compared with previous reports in literature (Xu et al., 2001, 2002; Essayagh et al., 2011; Alp et al., 2012). A. baumannii is a $\mathrm{G}^{-}$rod bacterium that is not fermented by carbohydrates, and is widely distributed in nature, hospital environments, and human skin. A. baumannii is a conditioned pathogen, $7 \%$ of which is located in human pharyngeal organs ( $\mathrm{Li}$ et al., 2005b). With the wide application of antibacterial drugs, bacterial drug resistance is sharply increasing and multi-drug resistant bacterial strains are evolving, causing great difficulties in clinical treatment. Hospital infections caused by $A$. baumannii are generally on the rise, and this is the main pathogen reported in some intensive care units (Pimentel et al., 2005). The infection rate of multi-drug resistant $A$. baumannii has drawn clinical attention (Oliveira and de Lencastre, 2002). Therefore, detailed knowledge of its distribution characteristics and dynamic changes in its drug resistance spectrum is important.

In this study, isolated $A$. baumannii showed a relatively low drug resistance rate to cefoperazone/sulbactam (5\%), but higher drug resistance rates to the other commonly used antibiotics, such as imipenem, which was $57 \%$ at XX hospital compared with less than $10 \%$ reported previously (Cheng et al., 2003). $\beta$-lactam antibiotics were highly resistant, whereby almost all of them exhibited $90 \%$ drug resistance. The drug resistance mechanism of $\mathrm{A}$. baumannii to $\beta$-lactam antibiotics mainly involves the production of $\beta$-lactamase. Acinetobacter could easily produce drug resistance by combining plasmids. A variety of resistant plasmids coexist, such as plasmid-mediated TEM-1 and TEM-2, TEM- $2 \beta$-lactamase, and chromosomemediated $\beta$-lactamase; the change in penicillin-binding proteins and the permeability decrease of outer membrane proteins can also result in drug resistance (Yang and $\mathrm{Li}, 2004$; $\mathrm{Yu}$ and $\mathrm{Li}$, 2006; Zhang and Chen, 2007). The data of the National Gram-Negative Resistance Survey indicated that from 1994 to 2001, P. aeruginosa ranked first among the infected $\mathrm{G}^{-}$bacteria in all hospitals; moreover, the sensitivities of the antibiotics declined (Wang and Chen, 2003).

In this study, the Gr rod bacteria had lower drug resistance to cefoperazone/sulbactam and piperacillin/tazobactam mainly because the antibiotics belonged to the restricted-use varieties in 
the unit, and were only used to treat severe infections with indications of bacterial drug sensitivity. In this study, the antibacterial drugs with a higher than $90 \%$ drug resistance rate of the detected $P$. aeruginosa accounted for $21.7 \%$, which is lower than results previously reported (Wei and Liu, 2006). Drugs with lower than 30\% resistance rates were only amikacin, aztreonam, piperacillin/tazobactam, cefepime, cefoperazone/sulbactam, ceftazidime, and imipenem, with drug resistance rates of $24.6,22.9,10.8,16.4,3.8,21.8$, and $22.6 \%$, respectively. The relatively low resistance level observed was related to the long-term and persistent use of the results of bacterial culture drug sensitivity tests in our burn ward to guide the clinical use of antibiotics and the strict antibiotic use system. None of the drugs listed above were clinical first-line medicines used in our department. With multi-drug resistance, P. aeruginosa could naturally resist multiple antibiotics and easily develop drug resistance in the course of antibiotic treatment. A variety of drug resistance mechanisms are known, including generation of $\beta$-lactamase, permeability decrease of the bacterial outer membrane, and changes in the structure and function of bacterial proteins (Hirakata and Izumikawa, 1998; Xu et al., 2001; Sun et al., 2009; Gu et al., 2005). Therefore, P. aeruginosa infections should be treated with reasonable use of antibiotics based on the epidemiological characteristics and bacterial culture results in this unit to avoid the generation of drug-resistant strains.

In this study, the detection rate of $S$. aureus in $\mathrm{G}^{+}$rod bacteria ranked first, accounting for $66.0 \%$. S. aureus is usually present in human skin or nasopharyngeal organs. $S$. aureus is the most common pathogen in festering infections that could cause all kinds of infections; it is particularly common in elderly and critical patients. MRSA infections are mainly associated with pneumonia, skin, or soft tissue infections, blood infections, and bone infections. MRSA is spread by direct or indirect contact with afflicted patients. MRSA was first identified in Britain in 1961. The clinical detection rate of MRSA annually increased in a span of 50 years. The extensive application of broad-spectrum antibiotics and the abuse of antibiotics have made MRSA an important pathogen in hospital infections. The harm caused by MRSA infections has drawn increasing clinical attention. In addition, the drug resistance of MRSE should not be ignored. This study showed that antibacterial drugs with a higher than $90 \%$ drug resistance rate to MRSA and MRSE accounted for 72.2 and $38.9 \%$, respectively; those with drug resistance rates to MRSA lower than 30\% included chloramphenicol, teicoplanin, and vancomycin, whereas those with drug resistance rates to MRSE lower than 30\% included only teicoplanin and vancomycin. In this study, Enterococcus was found to be resistant to the drugs teicoplanin and vancomycin, but with lower drug resistance rates, which may be related to the VanC type gene (Qian and Ning, 2011).

In view of the increase in bacterial drug resistance, attention should be given to the structural changes of pathogenic bacteria in the environment. Clinicians should focus closer attention to the local flora changes in this unit and consider future developmental trends to improve the activity and predictability of clinical anti-infection treatment. Bacterial culture susceptibility indicators are still important bases for the selection of antibiotics, and therefore, in the anti-infection treatment of burns. The specifications of antibiotics should be strictly implemented to use drugs appropriately.

\section{ACKNOWLEDGMENTS}

Study supported by the Medical Fund of Baotou, Inner Mongolia Province (\#2006-49). 


\title{
Conflicts of interest
}

\author{
The authors declare no conflict of interest.
}

\section{REFERENCES}

Alp E, Coruh A, Gunay GK, Yontar Y, et al. (2012). Risk factors for nosocomial infection and mortality in burn patients: 10 years of experience at a university hospital. J. Burn. Care Res. 33: 379-385.

Cheng Z, Zeng W and Jia W (2003). The resistance mechanism of Acinetobacter baumannii. World Notes Antibiot. 24: 63-64.

Essayagh T, Zohoun A, Essayagh M, Elameri A, et al. (2011). Bacterial epidemiology in the burns unit at military teaching hospital Mohamed V of Rabat. Ann. Biol. Clin. 69: 71-76.

Gu Y, Luo YP, Zhang WL and Zhang JM (2005). Imipenem-resistant Pseudomonas aeruginosa and detection of metalloB-lactamase. Chin. J. Nosocomiol. 3: 108-110.

Hirakata Y and Izumikawa K (1998). Rapid detection and evolution of clinical characteristics of emerging multiple-drugresistant Gram-negative rods carrying the metallo- $\beta$-lactamaes gene bla IMP. Antimicrob. Agents Chemother. 42: 2006-2011.

Li JT, Li Y and Qi HM (2005a). 2003-2003 Bacterial resistance surveillance study on Gram-negative bacilli in China. Chin. J. Lab. Med. 28: 19-29.

Li SL, Li Y and Hua C (2005b). Multidrug-Resistant Acinetobacter baumannii: current status and clinical countermeasures. Chin. J. Nosocomoiol. 15: 1438-1440.

Oliveira DC and de Lencastre H (2002). Multiplex PCR strategy for rapid identification of structural types and variants of the mec element in methicillin-resistant Staphylococcus aureus. Antimicrob. Agents Chemother. 46: 2155-2161.

Pimentel JD, Low J, Styles K, Harris OC, et al. (2005). Control of an outbreak of multi-drug-resistant Acinetobacter baumannii in an intensive care unit and a surgical ward. J. Hosp. Infect. 59: 249-253.

Qian C and Ning XW (2011). Detection of enterococci resistence and vancomycin resistant gene. J. Med. Sci. Cent. South China 39: 422-425.

Sun GQ, Zhang XM, Lan HL, Lu LF, et al. (2009). Pseudomonas aeruginosa isolation and resistance status analysis of infectious patients in intensive care unit. J. Pathogen Biol. 4: 608-610.

Wang $\mathrm{H}$ and Chen MJ (2003). Changes of antimicrobial resistance among nonfermenting gram-negative bacilli isolated from intensive care units from 1994 to 2001 in China. Zhonghua Yi Xue Za Zhi 83: 385-390.

Wei DN and Liu J (2006). Investigtion on bacterial flora and analisis of their antibiotic resistance from burn patients. Chin. J. Burns 22: 92-95.

Xiao GX (2004). Two problems about nosocomial infection in burn ward. Chin. J. Burns 20: 4-5.

Xu X, Zhang M and Liao P (2001). Analysis of bacterial resistance to antibiotics in a burn ward. Zhonghua Shao Shang Za Zhi 17: 83-87.

Xu Y, Li T, Qi S, Shen R, et al. (2002). An investigation of bacterial epidemiology and an analysis of bacterial resistance to antibiotics in a burn unit from 1993 to 1999. Zhonghua Shao Shang Za Zhi 18: 159-162.

Yang XJ and Li XY (2004). Advances in studies of mechanisms of anti-drug resistance of amotile bacilli against $\beta$-lactam antibiotics. Foreign Med. Sci. Section Clin. Biochem. Lab. Med. 25: 42-44.

Yu H and Li Q (2006). Drug-resistant analysis of 723 strains Staphylococcus epidermis. J. Clin. Transfus Lab. Med. 8: 35-36.

Zhang WY and Chen Q (2007). Epidemiological study of nosociomial infection by Acinetobacter spp. and the resistance of Acinetobacter baumannii. J. Trop. Med. 7: 1136-1138. 